\title{
Ameloblastic Carcinoma-Secondary Type (Dedifferentiated), Intraosseous
}

National Cancer Institute

\section{Source}

National Cancer Institute. Ameloblastic Carcinoma-Secondary Type (Dedifferentiated), Intraosseous. NCl Thesaurus. Code C54298.

A very rare ameloblastic carcinoma that originates from a pre-existing intraosseous benign ameloblastoma. 